\title{
Asymmetric nuclear reprogramming in somatic cell nuclear transfer?
}

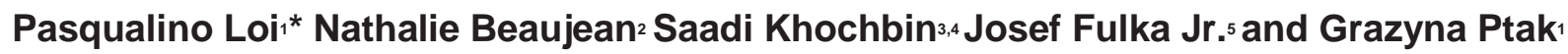 \\ 1Department of Comparative Biomedical Sciences, Teramo, Italy. \\ 2Biologie du De'veloppement et Reproduction, INRA, Jouy-en-Josas, France. \\ 3INSERM, U823, Institut Albert Bonniot, Epigenetic and Cell Signalling Team, Grenoble, France. \\ 4 Universite' Joseph Fourier, Grenoble, France. \\ 5Institute of Animal Sciences, Prague, Czech Republic \\ Funding agency: Research in PL and GP laboratories is founded by the European Science Foundation \\ EUROCORES Programme Euro-STELLS, contract no. ERAS-CT-2003-980409 and PRIN 2006. S.K. \\ laboratory is supported by grants from the Regulome Consortium (ANR-05-BLAN-0396-04), the CLARA \\ (EpiPro program) and ARECA (ARC) programmes. J. F. Jr acknowledges the Grant Sponsor MZe \\ 0002701401.
}

${ }^{*}$ Correspondence to: Pasqualino Loi, Department of Comparative Biomedical Sciences, University of Teramo, Piazza A. Moro 45, Teramo, TE 64100, Italy. E-mail: ploi@unite.it

\section{Summary}

Despite the progress achieved over the last decade after the birth of the first cloned mammal, the efficiency of reproductive cloning remains invariably low. However, research aiming at the use of nuclear transfer for the production of patient-tailored stem cells for cell/tissue therapy is progressing rapidly. Yet, reproductive cloning has many potential implications for animal breeding, transgenic research and the conservation of endangered species. In this article we suggest that the changes in the epi-/genotype observed in cloned embryos arise from unbalanced nuclear reprogramming between parental chromosomes. It is probable that the oocyte reprogramming machinery, devised for resident chromosomes, cannot target the paternal alleles of somatic cells. We, therefore, suggest that a reasonable approach to balance this asymmetry in nuclear reprogramming might involve the transient expression in donor cells of chromatin remodelling proteins, which are physiologically expressed during spermatogenesis, in order to induce a male-specific chromatin organisation in the somatic cells before nuclear transfer.

Abbreviations: NT, nuclear transfer; SCNT, somatic cell nuclear transfer; ES, embryonic stem cells; DNMT, de novo methylTrasferase; ICM, inner cell mass; TE, trophectoderm; 5 Aza, 5-azacitydin; TSA, tricostatin A; BRDT, bromodomain testis specific.

\section{Introduction}

Nuclear transfer (NT) in amphibians was devised to address the issue of nuclear equivalence during cell differentiation,(1) while its application in mammals first using embryonic,(2) then somatic cells(3) (somatic cell nuclear transfer, SCNT), was essentially finalised to the conservation (cloning) of highly valuable genotypes in animal breeding. The production of the first cloned sheep(3) had a domino effect worldwide with a large number of leading laboratories trying to optimise the cloning procedures. Such a massive effort has led to remarkable achievements, witnessed by the successful cloning of 11 mammalian species.(4) Meanwhile, the manipulation procedures for SCNT have been simplified, allowing the reconstruction of larger number of embryos,(5) with the realistic perspective of achieving the automation of the entire procedure in a short period of time.(6) Altogether, these improvements, somehow, compensate for the low efficiency of the procedure, thus, allowing the reliable production of cloned offspring, especially in cattle.(7)

However, despite our expanding knowledge about the mechanisms affecting nuclear reprogramming,(8) all the problems recorded from the beginning of the cloning era, such as foetal losses, $(9,10)$ postnatal mortality $(11)$ and thestill disputed—reduced life span of cloned animals(12,13) still prevent its full application.

In this hypothesis article, we suggest that the problems observed in cloned embryos might arise, at least in part, from an unbalanced nuclear reprogramming between parental chromosomes due to the fact that the oocyte reprogramming machinery cannot target the paternal genome of the somatic cells used as donors. We, therefore, suggest that chromatin remodelling proteins, which are physiologically expressed during spermatogenesis, should be transiently expressed in donor cells in order to induce a male-specific chromatin organisation in the somatic cells before nuclear transfer and to balance the asymmetry in nuclear reprogramming.

\section{Why is nuclear transfer so important? Applications of nuclear transfer}

NT was conceived as an experimental tool to demonstrate nuclear equivalence between zygotic nuclei and differentiated cells in early amphibian experiments,(14) and still represents a unique instrument for studying developmental biology and the epi-/genetic mechanisms underlying cellular differentiation, aging and cancer development.(4)

However SCNT is not only an experimental tool; it enables the clonal expansion of valuable meat- and milkproducing animals, and its potential in animal breeding has already been convincingly demonstrated in cattle.(15) Moreover, the recent approval by US Food and Drug Administration (FDA) of foodstuff from clones for human 
consumption(16) is a positive signal that will help overcoming the concerns raised about the quality of products derived from SCNT-produced animals. Moreover, the incorporation of SCNT in conservation projects could help prevent the decline of certain species of large mammals.(17,18)

The lack of embryonic stem cells in large animals has hindered the production of farm transgenic animals. SCNT, through the use of genetically transformed cells as donor nuclei, has partially overcome this limitation, and remarkable results have been already achieved in the production of transgenic farm animals.(19) Among the mostcommon agricultural applications of transgenic research, we can list improvement of carcass composition, lactation performance and wool production, although it is unlikely that these genetically modified products will meet commercial and social acceptance. Instead, farm animals that have been genetically modified for enhanced resistance to diseases and reduced environmental impact will be more easily accepted.(20)

Genetically modified animals produced by SCNT are also important in human medicine. The use of transgenic animals as sources of biologically active proteins(20) or as donors in xenotransplantation(21) is well documented. Certainly, the major biomedical indication for SCNT is the production of patient-tailored stem cells for cell/tissue therapy.(22,23) This last field of research, driven by the social relevance of the degenerative diseases that might potentially benefit from cell/tissue therapy, is progressing rapidly. The potential of SCNT for cell/tissue therapy, originally defined as "therapeutic cloning", has been demonstrated by the treatment of a genetic disease in an animal model by integrating nuclear transfer, gene therapy and stem cell biology.(24) However, "therapeutic cloning" implies the destruction of cloned embryos for stem cell derivation, with unavoidable ethical concerns.

Attempts to by-pass this ethical hurdle stimulated alternative, "embryo-free", approaches for nuclear reprogramming. Alternative routes to confer multipotency to differentiated cells led to the production of hybrids between somatic and stem cells(25,26) and the exposure of somatic cells to cell extracts.(27) The advantages and limits of these approaches are discussed in a recent review.(28) A more-sophisticated solution could be the use of "altered NT", where the downregulation of a critical gene for trophoblastic function in the nucleus before NT does not allow implantation of the reconstructed embryos to take place.(29) Efforts have also been made to characterise nuclear reprogramming in somatic cells more fully. The strongest evidence for a "controlled" nuclear reprogramming has recently been provided by a paper where the authors were able to differentiate foetal and adult fibroblasts into pluripotent cells by transfecting them with only four genes, OcT-4, Sox 2, c-Myc, and Klf4 that were selected from a panel of 24 candidate genes expressed in totipotent cells.(30) The pluripotency of these cells, named iPS cells (for inducible Pluripotent Stem cells), was assessed by functional assays, such as the formation of teratomas, embryoid bodies and chimeras, and by gene expression analysis. Although several pitfalls were reported, such as limited colonisation by iPS cells of the chimeras, and clone-to-clone variability in the gene expression profiles, probably due to a limited reprogramming or abnormal methylation of key promoter genes inOCT3/4, the most -recent work by the same authors,(31) and by two other independent groups, has now clearly demonstrated that iPS are similar to bona fide ES cells by all known criteria.(32,33)

This short overview provides compelling evidence on the overwhelming progress achieved on the direct reprogramming of somatic cells, a fundamental breakthrough in therapeutic cloning for stem cell therapy. However, in reproductive cloning improvements are not so evident despite the fact that reproductive cloning has many potential implications in animal breeding,(34) in the production of transgenic animals(35) and as a tool for conservation efforts. $(17,18)$ Therefore, the development of efficient nuclear reprogramming protocols for the production of healthy cloned animals is central for reproductive physiologists

\section{Reproductive cloning: current state of the art}

Owing to the progress accomplished in the last 10 years, we may assume that all mammalian species can be cloned, using most, if not all, cell types as nuclear donors. However, cloning outcomes are still low in all species tested so far.

Several factors account for this low efficiency. NT is a complex multi-step procedure, which includes oocyte maturation, enucleation, cell fusion/injection, oocyte activation and embryo culture, and the efficiency achieved in each one will account for the final success. Oocyte physiology, activation dynamics and pre-implantation embryo metabolism differ markedly among species, therefore, there is no unique, standardised protocol that can be applied, and species specific differences in cloning efficiency do exist. However, we believe that the species-specific issues have only a minor influence on cloning outcomes, because even in the most-suitable and most-studied species for SCNT, the bovine,(7) the efficiency is still very low. Rather, it is widely accepted that the main factor responsible for the demise of clones is the oocyte's failure to confer to the transplanted nucleus a full totipotent state.

\section{Nuclear reprogramming: resetting a somatic cell}

The paper on the cloning of Dolly was accompanied by the idea that nuclear quiescence (i.e. Go phase of the cell cycle), induced by serum starvation, was essential to obtain complete nuclear reprogramming(3,36) of the donor somatic cells. The second cloning paper suggested that the key factor for nuclear reprogramming was the prolonged permanence of the condensed chromosomes of the donor cell in the cytoplasm of unactivated, enucleated oocytes.(37) Both hypotheses have been supported by scientific evidence, as transcription factors are transiently displaced from the chromosomes in both conditions, $(38,39)$ thus, theoretically, facilitating nuclear reprogramming. In practice, however, despite the fact the Go stage can be uniformly induced in a cell population, and that the exposure period of chromosomes in an unactivated oocyte is easily controllable, only a minority of somatic nuclei can be completely reprogrammed. Moreover, the "reprogrammability" of the nucleus of donor cells does not seem to be 
strictly linked to their cell cycle phase.(40,41) although the cell cycle phase of the donor nucleus, when transferred into a metaphase II oocyte, can have an effect on the ploidy of the reconstructed embryo.(42)

A third hypothesis, proposed by a group of researchers, who were sceptical about the possibility of producing viable clones by NT of somatic cells, suggested that Dolly was not derived from terminally differentiated cells, but from a less differentiated subset of progenitor/stem cells that are more amenable for reprogramming and are present in any cell population.(43) The hypothesis that adult stem cells might undergo nuclear reprogramming more easily is under scientific scrutiny with, so far, controversial findings. The efficiency of nuclear reprogramming in stem cells isolated from different adult tissues or in differentiated, somatic cells was assessed by following the derived embryos during early development or up to term. In these experiments, comparable results were obtained(44-46) with both types of cells. Furthermore, among the studied adult stem cells marked variations in the ability to produce cloned offspring were observed, probably as a result of the different epigenetic/genetic status of these cells.(47)

In contrast, mature mice B and T lymphocytes,(48) and postmitotic olfactory neurons,(49) which are somatic cells clearly terminally differentiated, have been used for NT. Live offspring have been obtained from olfactory neurons and B lymphocytes, but only through a modified two-step NT procedure, where ES cells derived from the cloned blastocysts were injected into tetraploid blastocysts and then transferred into foster mothers for development to term. This "twostep nuclear transfer" was adopted because no viable pups were obtained from standard SCNT embryos in preliminary experiments. Therefore, these achievements might be interpreted as a sort of "compromise" solution, for tetraploid embryo complementation actually skips the need for a functional placenta, which is know to be the main limit of SCNT CLONES.

So far no major study has been carried out on how nuclear reprogramming is regulated and on its consequences. An overview of the literature indicates that epigenetic deregulation and abnormal gene expression in pre- and postimplantation embryos,(9,53,54) newborn animals and extraembryonic tissues(55) are commonly found in cloned animals. The persistence of a donor-specific de-regulation in gene expression in SCNT embryos, before and after implantation, demonstrates that the epigenetic memory of the donor nuclei is not reset by the oocyte.(56) In addition, SCNT early embryos fail to reactivate key genes essential for early development(57) and for the first steps of ontogenesis.(58)

The gene expression patterns of cloned embryos are currently under the spotlight, and the high-throughput gene expression analysis is giving precious information about the gene expression profiles of individual SCNT embryos.(59,60) Surprisingly, the results are rather promising, since they indicate that SCNT does not have much effect on gene expression as monitored in high-throughput gene expression analyses.(61) Moreover, a recent report ascribes a major effect on gene expression to the culture system, rather than to the SCNT procedure per se.(62)

A general summary of the published data suggests that, while major alterations are detected in the global epigenetic reorganization of SCNT embryos, namely changes in DNA methylation,(63) the analyses carried on the transcriptome are indicative of a consistent reprogramming following SCNT.

These data are encouraging, although in conflict with the severe phenotype observed in foetuses and extraembryonic tissues in embryos, and they are valuable as they suggest which molecular alterations should be targeted to improve nuclear reprogramming. For example, a finding common to all studies on the epigenetic status of the SCNT embryos is the higher methylation levels in early embryos, (63) with the apparent exception of the pig.(64)

DNA methylation in early embryonic development across species and in SCNT embryos Addition of a methyl group to $\mathrm{CpG}$ islands marks genes that have to be repressed. DNA methylation, together with Polycomb protein complexes, is stably maintained during cell proliferation and is responsible for the cellular memory.(65) The global methylation pattern of somatic cells is rather static, the only exceptions being the ageing process and cancer transformation. (65) On the contrary, the early Figure-1-upper panel embryo undergoes dynamic chances in its methylation profiles. Upon fertilization, an asymmetric DNA demethylation takes place, as the paternal pronucleus is actively demethylated during the first cell cycle, while the maternal one is unaffected.(66) A passive demethylation of the maternal and paternal genomes follows during the early cleavages. $(63,68)$ This is caused by the nuclear exclusion of the DeNovo MeThyltrasferase 10 (DNMT10), the oocyte variant of DNMT1, the enzyme that puts methyl groups on DNA.(67) At the blastocyst stage, a progressive methylation is observed in concomitance with the commitment of cells to two lineages: the Inner Cell Mass, (ICM) and the trophoectoderm, (TE).(69) In the mouse, TE cells are hypomethylated in comparison to ICM cells and this may be linked to the limited life span of the mouse extraembryonic tissues.(70) This hypothesis is supported by the heavier methylation marks observed in TE from human(71) and cattle embryos,(72) where gestation is longer and, therefore, the placenta has a longer lifespan. DNA methylation is a landmark of epigenetic regulation and, therefore, should be considered a highly conserved mechanism among mammals. Unexpectedly, studies on different mammalian embryos unveil remarkable species specific differences in methylation patterns. Rat, pig and bovine, like the mouse, show active demethylation of the paternal pronuclei in the zygote, followed by passive demethylation during early cleavages and asymmetric methylation of the TE and ICM cell lineages-although with quantitative and temporal differences among species.(63,69) In contrast, in sheep and rabbit, the paternal pronucleus does not lose its methylation marks after fertilisation, and the paternal and maternal genomes are not passively demethylated during the early cleavages.(73,74) Human zygotes are somehow in the middle, as asymmetric demethylation of one of the two pronuclei is observed in only half of the processed zygotes, while, in the other half both maternal and paternal pronuclei still show a faint methylation.(71) It is indeed difficult to draw precise conclusions from these data. In our interspecific Intra Cytoplasmic Sperm Injection (ICSI) study, we demonstrated, on one hand, that the paternal genome of species that are resistant to demethylation (i.e. sheep) can be demethylated in mouse oocytes; on the other hand, in sheep oocytes, the paternal genome coming from mouse sperm can be only partially 
demethylated.(75) These data suggest that both the oocyte cytoplasmic environment and the paternal chromatin composition/structure are important for reprogramming, as far as DNA methylation is concerned.

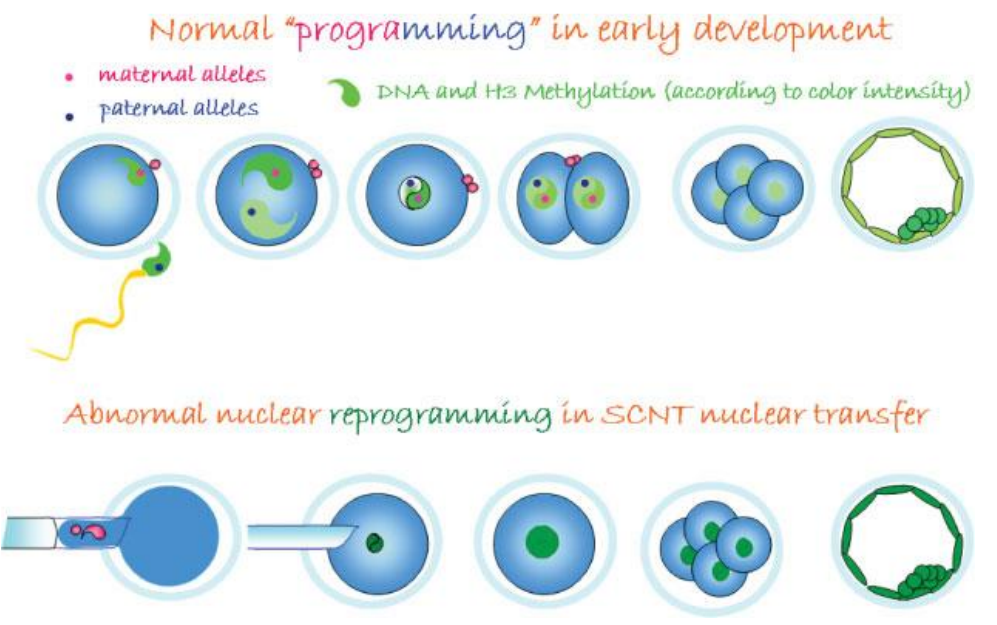

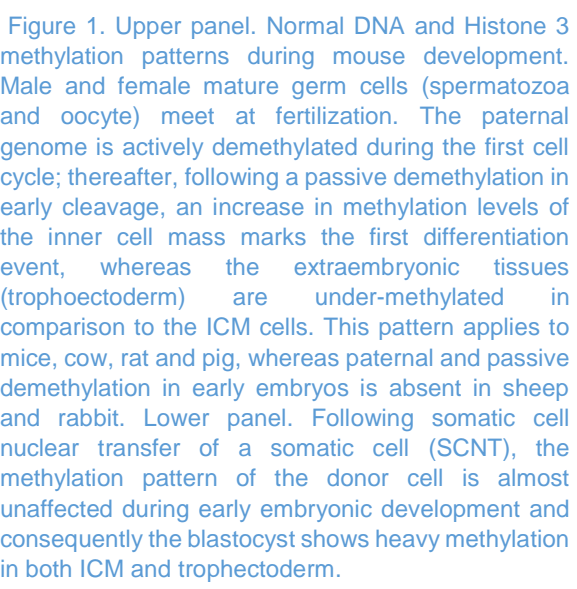

in both ICM and trophectoderm.

The finding that SCNT embryos have higher DNA methylation levels than normal embryos Figure-1-lower panel might be explained by the persistence of somatic methylation marks during reprogramming. For this reason, one approach suggests treating donor somatic cells with demethylation agents, mainly 5-Azacytidine (5-Aza),(76) or histone deacethylase inhibitors, like Trichostatin A (TSA), (77) before nuclear transfer. The rationale behind this approach is to remove repressive methylation marks (with 5-Aza), or to confer to the chromatin an "open" structure, more accessible to reprogramming factors (with TSA). However, very few cloned bovine embryos derived from 5Aza-treated cells reach blastocyst stage.(76) This finding is not surprising. It is known that 5-Aza increases the "stickiness" of chromosomes, leading to massive DNA rearrangement and micronuclei formation,(78) thus magnifying the intrinsic drift of SCNT-cloned embryos toward aneuploidy.(74) More promising is the use of TSA. Several reports have already been published in which the development to blastocyst stage in cattle(76) and to term in mice following TSA treatment of donor cells was analysed.( 77,79$)$ TSA increased the efficiency of reprogramming up to the blastocyst stage in both cattle and mice, and, in the latter, it induced a 5-fold increase in the efficiency of the cloning outcome in terms of live offspring.(79) In addition, TSA treatment of donor cells was crucial for the successful cloning by SCNTof an outbred mouse strain, which has been known to be resistant to nuclear reprogramming.(80,81)

\section{Asymmetry in nuclear reprogramming?}

ES cells are the best candidates for NT; however, this applies to mouse only, for ES are not yet available in large animals. The improvements in nuclear reprogramming efficiency obtained with TSA, a histone deacetylase inhibitor, are indeed promising, but it needs to be tested in species other than the mouse. Furthermore, TSA exerts a bulk, unspecific action, and, therefore, negative epigenetic changes can not be excluded.

Thus, at the moment, there is little indication on how nuclear reprogramming might be improved. However, some suggestions might come from a pilot experiment looking at differences in global methylation profiles in normal, invitro-fertilized, SCNT-derived, androgenetic and parthenogenetic sheep embryos (Loi P et al. unpublished). This preliminary study shows striking similarities between SCNT-derived and diploid androgenetic embryos as, in both of them, ICM and TE cells are heavily methylated, whereas TE cells of normal embryos are largely demethylated. This finding led us to ask whether the oocyte may not be (fully) capable of reprogramming the paternal genome. This hypothesis is also supported by the observation that extraembryonic tissues, which derive from the TE cells and in which the paternal alleles of imprinted genes are expressed, are frequently abnormal in SCNT embryos (813)

Recently, a demethylation activity was demonstrated by functional assays (i.e. nuclear and DNA transfer) in Xenopus oocytes, which could help understand the mechanism leading to the reactivation of OCT4, the mostcharacterised pluripotency marker, in somatic donor cells.(82) Re-activation of OCT4in the nuclei of terminally differentiated thymus cells was observed when they were injected into Germinal Vesicles (Xenopus oocytes).(83) Reactivation of the OCT4 gene is probably preceded by extensive nuclear remodelling of injected intact nuclei, because de-proteinized genomic and also plasmid DNAs are expressed with a progressively shorter delay. The demethylase activity was also found to be highly selective, acting only on the promoter, and not on the enhancers of OCT4.(82)

It is therefore likely that the oocyte demethylase, released in the cytoplasm upon meiotic progression, might be responsible for the reprogramming of somatic nuclei. It is also plausible that such oocyte-specific demethylase, 
originally designed to target maternal chromosomes during oocyte preparation for development, recognizes only the maternal alleles of the somatic cell nucleus, leaving the paternal ones unaffected (Fig. 2, upper panel). Therefore, it is possible that the phenotypic/epigenetic abnormalities and the low efficiency of SCNT are caused by an "asymmetric" reprogramming, where the paternal genome is largely unaffected by the reprogramming machinery, or alternatively, by the impossibility of re-establishing the physiological epigenetic asymmetry typical of pre-implantation development.(84)
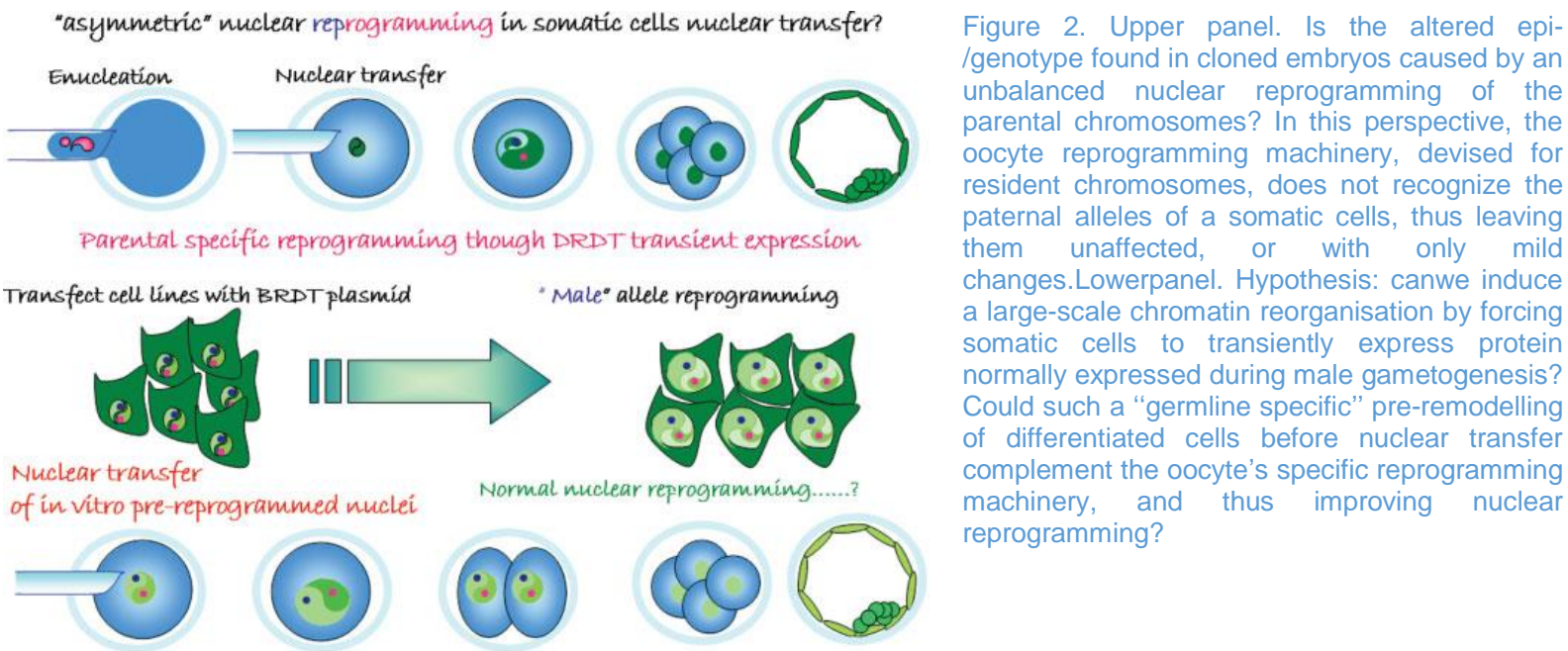

Indeed, the following are evidence for the existence of a physiological epigenetic asymmetry between parental genomes.

1. The parental sets of chromosomes are spatially separated during early embryogenesis in mouse, $(85,86)$ suggesting a sex-dependent chromatin remodelling.

2. Oocyte chromosomes are completely reprogrammed-or better "programmed"-for development by the end of the maturation process as a result of epigenetic modifications taking place in the germinal vesicle. This notion is supported by the lack of major DNA modifications at the beginning of embryogenesis, and the high frequency of development to the blastocyst stage—similar to, or even higher than in control IVF embryos_of artificially activated mouse oocytes.(87-89) These diploid parthenogenetic blastocysts implant at very high rates and undergo normal organogenesis, and their developmental block is not the consequence of incomplete reprogramming, rather it is due to the absence of a paternal set of chromosomes.(90)

3. TSA facilitates nuclear reprogramming through the inhibition of histone deacethylases (HDACH). The resulting hyperacethylated nucleosomes confer an "open" structure on the chromatin, which may in turn facilitate its accessibility to the reprogramming machinery. There is evidence of a paternal chromatin competition for the hyperacetylated pool of maternal Histone 4,K18 with only the paternal pronucleus positive for acetylated H4 (acH4K18) before $S$ phase in mouse zygotes.(91,92) Therefore, we can speculate that the paternal alleles of the transplanted somatic nuclei preferentially benefit from the TSA treatment in the post-activation window, thus justifying the improved reprogramming described.(79,80)

4. Allele-specific chromatin organisation detected by high throughput genome-wide profiling of histone modifications suggests an allele-specific read-out of transcription factors and of the reprogramming machinery.(93)

If we then assume that the "asymmetry" in the parental genomes is real, does it mean that we need to remodel a somatic cell according to what occurs in germline cells?

During male germ-cell maturation, chromatin structures undergo dramatic changes (Figure 3) leading to the exchange of somatic histones with sperm-specific protamines.(94) The postmeiotic massive histone replacement and the associated global genome reorganisation are directed by mechanisms that remain completely unknown. Current knowledge implies a stepwise replacement of histones by transition proteins and finally protamines to pack the mature spermatozoid's genome. Recent investigations highlight, however, the existence of another level of organisation involving new testis-specific histone variants. These proteins accumulate essentially during late spermiogenesis and are stably associated with the genome of mature spermatozoa. They, therefore, possess the potential to specify particular regions of the male germ cell genome and could contribute to the transmission of a new type of epigenetic information to the egg. It has also been shown that these late events follow a general genome reorganisation triggered by a massive wave of histone acetylation occurring at the beginning of the spermatid elongation process. One of the most spectacular consequences of this phenomenon is the re-programming of pericentromeric heterochromatin, which, from a somatic-type structure, known as chromocenter, evolves towards a 
new type of structure that accumulates hyper-acetylated histones just before their replacement by the new histone variants.(95)
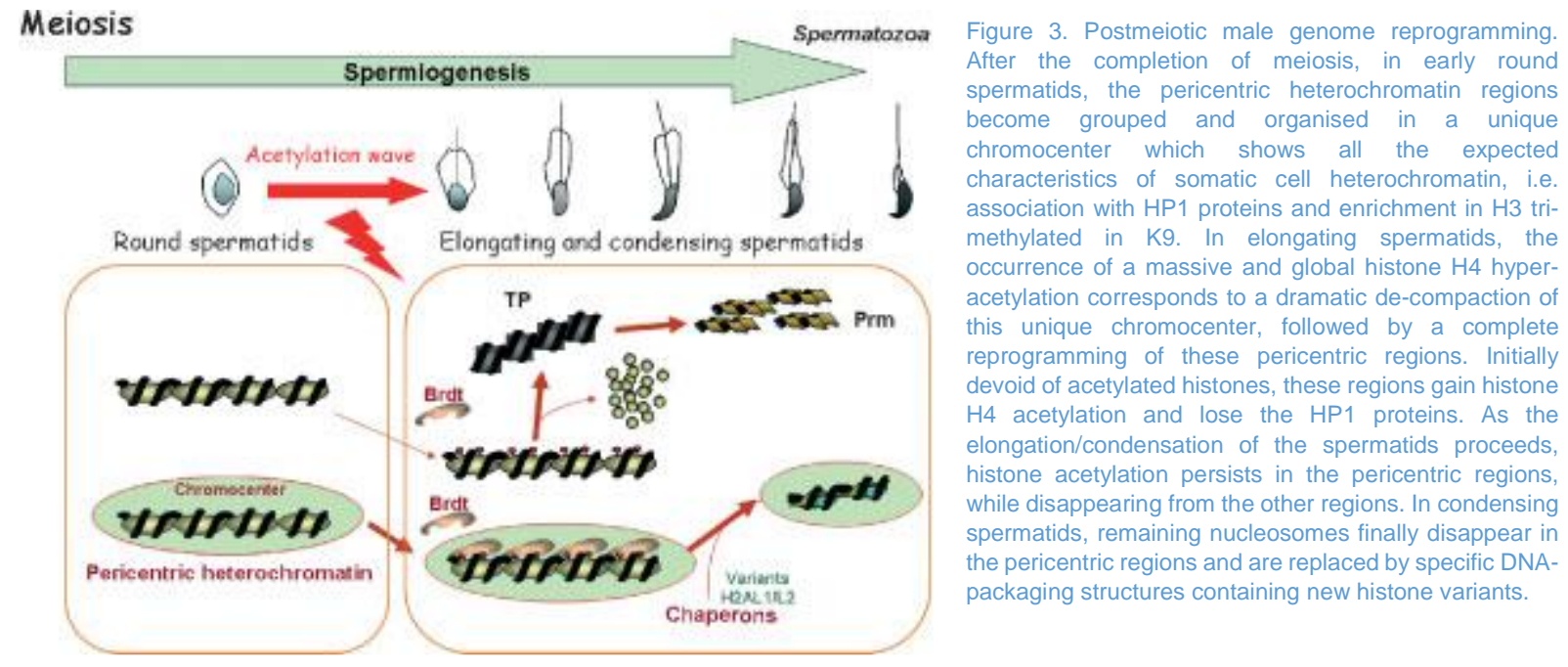

Testis-specific factors, which interact exclusively with acetylated chromatin, are therefore good candidates for directing the genome reprogramming events triggered by the wave of histone hyperacetylation. Interestingly, one of these factors, the BRomo Domain Testis-specific (BRDT) protein, can induce chromatin remodelling in a histone acetylation dependent manner in isolated nuclei and also in living somatic cells expressing BRDT.(96)

These observations might have relevant implication for the development of efficient in vitro "pre-reprogramming" of somatic cells prior to nuclear transfer. Indeed, the largescale chromatin reorganisation brought upon by exogenous expression in donor somatic cells of BRDT, a factor specifically expressed during male gametogenesis, might be used to induce a "germ-line-specific" pre-remodelling of these cells, which might complement the oocyte's specific reprogramming, and improve nuclear reprogramming in SCNT (Fig. 2, lower panel). This strategy raises obvious concerns on the effects resulting from the expression of a transgene-in this case a paternal gene-during early embryogenesis and differentiation. There are several ways to get around this problem. The treatment of permeabilised somatic cells with BRDT results in a reprogramming similar to that in cells expressing a BRDT transgene.(96) Since heat-denaturated (55 and 758C) cells have already been successfully used for nuclear transfer leading to the production of healthy living lambs,(97) the use of BRDT-treated permeabilised cells could also be envisaged. However, since denatured membranes cannot be fused, nuclear transfer will have to be done by direct injection of the nuclei. Therefore, when cell fusion is the only option for nuclear transfer (for example, due to the size of the donor cells), then alternative approaches should be pursued, such as use of adenovirus,(98) or highthroughput screening of small molecule libraries for the identification of synthetic analogues that can replace BRDT and that could be loaded into the cells by electroporation or streptolisin O.

\section{Conclusions}

The reversal of cell differentiation through cloning is a highly complex process whose outcome is impossible to predict. Actually, it is rather surprising that nuclear transfer works at all, given the dramatic shortcuts that we are using in order to try to recapitulate in few cell cycles the pan-epigenetic reprogramming that takes place separately in the two germ cells over a period of several weeks to months according to the species. Perhaps, we nurtured the misleading perception that a single breakthrough would overcome all the hurdles in SCNT. Improvements in the outcome of SCNT will be rather achieved by working at different levels, starting from a clearer definition of the gamete/embryo metabolic needs in vitro, and ending with a better controlled nuclear reprogramming. Regarding the first issue, there is ample margin for improvement. For instance, early mouse embryos cloned from myoblasts develop better in media specific for myoblast primary culture than in standard embryo culture media,(99) suggesting that SCNT embryos might have a more somatic-like metabolism. The characterisation of which media are more suitable for the culture of SCNT embryos might improve their metabolism and homeostasis, with obvious benefits on the genome remodelling and finally on their survival.

The control of nuclear reprogramming is certainly more complicated. The severity of abnormal phenotypes in SCNT clones is the consequence of bypassing the epigenetic formatting that normally takes place during male and female gem cell development and maturation. This issue is important especially for the paternal genome of the donor cell, as the maternal one is replaced back into its original reprogramming milieu. The induction of a sex-specific parental reprogramming, through the transient expression of one or more remodelling factors that are normally expressed during male germ cell maturation, should in theory result in a better physiological chromatin remodelling and, in turn, improve nuclear reprogramming. 


\section{Acknowledgments}

\section{We thank Helena Fulka for critical reading of the manuscript.}

\section{References}

1. Gurdon JB, Uehlinger V. 1966. "Fertile" intestine nuclei. Nature 210: 1240-1241.

2. Willadsen SM. 1986. Nuclear transplantation in sheep embryos. Nature 320:63-65

3. Wilmut I, Schnieke AE, McWhir J, Kind AJ, Campbell KH. 1997. Viable offspring derived from fetal and adult mammalian cells. Nature 385:810813.

4. Meissner A, Jaenisch R. 2006. Mammalian nuclear transfer. Dev Dyn 235:2460-2469.

5. Lagutina I, et al. 2005. Somatic cell nuclear transfer in horses: effect of oocyte morphology, embryo reconstruction method and donor cell type. Reproduction 130:559-567.

6. Vajta G. 2007. Handmade cloning: the future way of nuclear transfer? Trends In Biotechnology 25:250-253.

7. Tian XC, et al. 2007. Nuclear reprogramming by somatic cell nuclear transfer-the cattle story. Soc Reprod Fertil Suppl 64:327-339.

8. Tsunoda Y, Kato Y. 2002. Recent progress and problems in animal cloning. Differentiation 69:158-161.

9. Rideout WM III, Eggan K, Jaenisch R. 2001. Nuclear cloning and epigenetic reprogramming of the genome. Science 293:1093-1098.

10. Latham KE. 2005. Early and delayed aspects of nuclear reprogramming during cloning. Biol Cell 97:119-132.

11. Loi $P$, et al. 2006. Placental abnormalities associated with post-natal mortality in sheep somatic cell clones. Theriogenology $65: 1110-1121$.

12. Ogura A, et al. 2002. Phenotypic effects of somatic cell cloning in the mouse. Cloning Stem Cells 4:397-405.

13. Tamashiro KL, et al. 2003. Phenotype of cloned mice: development, behaviour, and physiology. Exp Biol Med 228:1193-1200.

14. Gurdon JB. 2006. From nuclear transfer to nuclear reprogramming: the reversal of cell differentiation. Annu Rev Cell Dev Biol 22:1-22.

15. Bousquet D, Blondin P. 2004. Potential uses of cloning in breeding schemes: dairy cattle. Cloning Stem Cells 6:190-197.

16. Yang X, et al. 2007. Risk assessment of meat and milk from cloned animals. Nat Biotechnol 25:77-83.

17. Loi P, et al. 2001. Genetic rescue of an endangered mammal by crossspecies nuclear transfer using post-mortem somatic cells. Nat Biotechnol 19:962-964.

18. Holt WV, et al. 2004. Wildlife conservation and reproductive cloning. Reproduction 127:317-324.

19. Robl JM, Wang Z, Kasinathan P, Kuroiwa Y. 2007. Transgenic animal production and animal biotechnology. Theriogenology 67:127-133

20. Niemann H, Kues WA. 2007. Transgenic farm animals: an update. Reprod Fertil Dev 19:762-770.

21. Prather RS, Hawley RJ, Carter DB, Lai L, Greenstein JL. 2002. Transgenic swine for biomedicine and agriculture. Theriogenology 59: $115-123$.

22. Trounson AO. 2006. Future and applications of cloning. Methods Mol Biol 348:319-332.

23. Pomerantz J, Blau HM. 2004. Nuclear reprogramming: a key to stem cell function in regenerative medicine. Nat Cell Biol 6:810-816.

24. Rideout WM 3rd, Hochedlinger K, Kyba M, Daley GQ, Jaenisch R. 2002. Correction of a genetic defect by nuclear transplantation and combined cell and gene therapy. Cell 109:17-27.

25. Tada M, Takahama Y, Abe K, Nakatsuji N, Tada T. 2001. Nuclear reprogramming of somatic cells by in vitro hybridization with ES cells. Curr Biol 11:1553-1558.

26. Taranger CK, et al. 2005. Induction of dedifferentiation, genome wide transcriptional programming, and epigenetic reprogramming by extracts of carcinoma and embryonic stem cells. Mol Biol Cell 16:5719-5735.

27. Hakelien AM, Kuntziger T, Gaustad KG, Marstad A, Collas P. 2006. In vitro reprogramming of nuclei and cells. Methods Mol Biol 348:259-368.

28. Hochedlinger K, Jaenisch R. 2006. Nuclear reprogramming and pluripotency. Nature 441:1061-1067.

29. Hurlbut WB. 2005. Altered nuclear transfer: a way forward for embryonic stem cell research. Stem Cell Rev 1:293-300.

30. Takahashi K, Yamanaka S. 2006. Induction of pluripotent stem cells from mouse embryonic and adult fibroblast cultures by defined factors. Cell 126:663-676.

31. Okita K, Ichisaka T, Yamanaka S. 2007. Generation of germlinecompetent induced pluripotent stem cells. Nature 448:313-317.

32. Wernig M, et al. 2007. In vitro reprogramming of fibroblasts into a pluripotent ES-cell-like state. Nature 448:318-324

33. Rossant J. 2007. Stem cells: the magic brew. Nature 448:260-262.

34. Wells DN. 2003. Cloning in livestock agriculture. Reprod Suppl 61:131-150.

35. Robl JM, Wang Z, Kasinathan P, Kuroiwa Y. 2007. Transgenic animal production and animal biotechnology. Theriogenology 67:127-133.

36. Campbell KH. 1999. Nuclear equivalence, nuclear transfer, and the cell cycle. Cloning 1:3-15.

37. Wakayama T, Perry AC, Zuccotti M, Johnson KR, Yanagimachi R. 1998. Full-term development of mice from enucleated oocytes injected with cumulus cell nuclei. Nature 394:369-374.

38. Withfield JF, Boynton AL, Rixon RH, Youdale T. 1985. The control of animal cell proliferation by calcium, Carp-calmodulin, and cyclic AMP. In: Boynton AL, Leffert HL, editors. Control of Animal Cell Proliferation, vol 1. London: Academic Press. pp 331-365.

39. Martı́nez-Balba's MA, Dey A, Rabindran SK, Ozato K, Wu C. 1995. Displacement of sequence-specific transcription factors from mitotic chromatin. Cell 83:29-38.

40. Wakayama T, Rodriguez I, Perry AC, Yanagimachi R, Mombaerts P. 1999. Mice cloned from embryonic stem cells. Proc Natl Acad Sci USA 96:14984-14989.

41. Cibelli JB, et al. 1988. Cloned transgenic calves produced from nonquiescent fetal fibroblasts. Science 280:1256-1258.

42. Campbell KH, Loi P, Otaegui PJ, Wilmut I. 1996. Cell cycle co-ordination in embryo cloning by nuclear transfer. Rev Reprod 1:40-46.

43. Sgaramella V, Zinder ND. 1998. Dolly confirmation. Science 279:635, 637-638.

44. Inoue K, et al. 2007. Differential developmental ability of embryos cloned from tissue-specific stem cells. Stem Cells [Epub ahead of print] PMID:1725551.

45. Mizutani E, et al. 2006. Developmental ability of cloned embryos from neural stem cells. Reproduction 132:849-857.

46. Inoue K, et al. 2006. Inefficient reprogramming of the hematopoietic stem cell genome following nuclear transfer. J Cell Sci 119:1985-1991.

47. Oback B, Wells DN. 2006. Donor cell differentiation, reprogramming, and cloning efficiency: Elusive or illusive correlation? Mol Reprod Dev 74: 646-654.

48. Hochedlinger K, Jaenisch R. 2002. Monoclonal mice generated by nuclear transfer from mature B and T donor cells. Nature 415:1035-1038.

49. Eggan K, et al. 2004. Mice cloned from olfactory sensory neurons. Nature 428:44-49.

50. Inoue K, et al. 2005. Generation of cloned mice by direct nuclear transfer from natural killer T cells. Curr Biol 15:1114-1148.

51. Rideout WM 3rd, et al. 2000. Generation of mice from wild-type and targeted ES cells by nuclear cloning. Nat Genet 24:109-110.

52. Wakayama T, Rodriguez I, Perry AC, Yanagimachi R, Mombaerts P. 1999. Mice cloned from embryonic stem cells. Proc Natl Acad Sci USA 96:14984-14989.

53. Kohda T, et al. 2005. Variation in gene expression and aberrantly regulated chromosome regions in cloned mice. Biol Reprod 73:1302-1311.

54. Cezar GG, et al. 2003. Genome-wide epigenetic alterations in cloned bovine fetuses. Biol Reprod 68:1009-1014.

55. Kremenskoy M, et al. 2006. DNA methylation profiles of donor nuclei cells and tissues of cloned bovine fetuses. J Reprod Dev 52:259-266.

56. Ng RK, Gurdon JB. 2005. Epigenetic memory of active gene transcription is inherited through somatic cell nuclear transfer. Proc Natl Acad Sci USA 102:1957-1962.

57. Boiani M, Eckardt S, Scholer HR, McLaughlin KJ. 2002. Oct4 distribution and level in mouse clones: consequences for pluripotency. Genes Dev 16:1209-1219.

58. Jouneau A, et al. 2006. Developmental abnormalities of NT mouse embryos appear early after implantation. Development 133:1597-1607.

59. Wuensch A, et al. 2007. Quantitative monitoring of pluripotency gene activation after somatic cloning in cattle. Biol Reprod 76:983-991.

60. Smith C, et al. 2007. Simultaneous gene quantitation of multiple genes in individual bovine nuclear transfer blastocysts. Reproduction 133:231242.

61. Smith SL, et al. 2005. Global gene expression profiles reveal significant nuclear reprogramming by the blastocyst stage after cloning. Proc Natl Acad Sci USA 102:17582-17587.

62. Somers J, et al. 2006. Gene expression profiling of individual bovine nuclear transfer blastocysts. Reproduction 131:1073-1084. 
63. Haaf T. 2006. Methylation dynamics in the early mammalian embryo: implications of genome reprogramming defects for development. Curr Top Microbiol Immunol 310:13-22.

64. Kang YK, et al. 2001. Typical demethylation events in cloned pig embryos. Clues on species-specific differences in epigenetic reprogramming of a cloned donor genome. J Biol Chem.276:39980-39984.

65. Bird A. 2002. DNA methylation patterns and epigenetic memory. Genes Dev 16:6-21.

66. Mayer W, Niveleau A, Walter J, Fundele R, Haaf T. 2000. Demethylation of the zygotic paternal genome. Nature 403:501-502.

67. Howell CY, et al. 2001. Genomic imprinting disrupted by a maternal effect mutation in the Dnmt1 gene. Cell 104:829-838.

68. Santos F, Peters AH, Otte AP, Reik W, Dean W. 2005. Dynamic chromatin modifications characterise the first cell cycle in mouse embryos. Dev Biol 280:225-236.

69. Morgan HD, Santos F, Green K, Dean W, Reik W. 2005. Epigenetic reprogramming in mammals. Hum Mol Genet 14:R47-R58.

70. Reik W, et al. 2003. Epigenetic asymmetry in the mammalian zygote and early embryo: relationship to lineage commitment? Philos Trans $R$ Soc Lond B Biol Sci 358:1403-1409.

71. Fulka H, Mrazek M, Tepla O, Fulka J Jr. 2004. DNA methylation pattern in human zygotes and developing embryos. Reproduction 128:703708.

72. Santos F, et al. 2003. Epigenetic marking correlates with developmental potential in cloned bovine preimplantation embryos. Curr Biol 13:11161121.

73. Beaujean N, et al. 2004. Effect of limited DNA methylation reprogramming in the normal sheep embryo on somatic cell nuclear transfer. Biol Reprod 71:185-193.

74. Shi W, Dirim F, Wolf E, Zakhartchenko V, Haaf T. 2004. Methylation reprogramming and chromosomal aneuploidy in in vivo fertilized and cloned rabbit preimplantation embryos. Biol Reprod 71:340-347.

75. Beaujean N, et al. 2004. The effect of interspecific oocytes on demethylation of sperm DNA. Proc Natl Acad Sci USA 101:7636-7640.

76. Enright BP, Kubota C, Yang X, Tian XC. 2003. Epigenetic characteristics and development of embryos cloned from donor cells treated by trichostatin A or 5-aza-20-deoxycytidine. Biol Reprod 69:896-901.

77. Rybouchkin A, Kato Y, Tsunoda Y. 2006. Role of histone acetylation in reprogramming of somatic nuclei following nuclear transfer. Biol Reprod $74: 1083-1089$

78. Kiziltepe T, et al. 2007. 5-Azacytidine, a DNA methyltransferase inhibitor, induces ATR-mediated DNA double-strand break responses, apoptosis, and synergistic cytotoxicity with doxorubicin and bortezomib against multiple myeloma cells. Mol Cancer Ther 6:1718-1728.

79. Kishigami S. 2006. Significant improvement of mouse cloning technique by treatment with trichostatin A after somatic nuclear transfer. Biochem Biophys Res Commun 340:183-189.

80. Kishigami S, et al. 2007. Successful mouse cloning of an outbred strain by trichostatin A treatment after somatic nuclear transfer. J Reprod Dev 53:165-170.

81. Wakayama T. Production of cloned mice and ES cells from adult somatic cells by nuclear transfer: how to improve cloning efficiency? J Reprod Dev. 2007 Feb; 53(1):13-26.

82. Simonsson S, Gurdon J. 2004. DNA demethylation is necessary for the epigenetic reprogramming of somatic cell nuclei. Nat Cell Biol 6:984990.

83. Byrne JA, Simonsson S, Western PS, Gurdon JB. 2003. Nuclei of adult mammalian somatic cells are directly reprogrammed to oct-4 stem cell gene expression by amphibian oocytes. Curr Biol 13:1206-1213.

84. Morgan HD, Santos F, Green K, Dean W, Reik W. 2005. Epigenetic reprogramming in mammals. Hum Mol Genet 14:R47-58.

85. Barton SC, et al. 2001. Genome-wide methylation patterns in normal and uniparental early mouse embryos. Hum Mol Genet 10:2983-2987.

86. Haaf T. 2001. The battle of the sexes after fertilization: behaviour of paternal and maternal chromosomes in the early mammalian embryo. Chromosome Res 9:263-271.

87. Kaufman MH, Barton SC, Surani MA. 1977. Normal postimplantation development of mouse parthenogenetic embryos to the forelimb bud stage. Nature 265:53-55

88. Kaufman MH, Sachs L. 1976. Complete preimplantation development in culture of parthenogenetic mouse embryos. J Embryol Exp Morphol 35: $179-190$.

89. Loi P, Ledda S, Fulka J Jr, Cappai P, Moor RM. 1998. Development of parthenogenetic and cloned ovine embryos: effect of activation protocols. Biol Reprod 58:1177-1187.

90. McGrath J, Solter D. 1984. Completion of mouse embryogenesis requires both the maternal and paternal genomes. Cell 37:179- 183.

91. van der Heijden GW, et al. 2005. Asymmetry in histone H3 variants and lysine methylation between paternal and maternal chromatin of the early mouse zygote. Mech Dev 122:1008-1022.

92. Yoshida N, Brahmajosyula M, Shoji S, Amanai M, Perry AC. 2007. Epigenetic discrimination by mouse metaphase II oocytes mediates asymmetric chromatin remodeling independently of meiotic exit. Dev Biol 301:464-477.

93. Mikkelsen TS, et al. 2007. Genome-wide maps of chromatin state in pluripotent and lineage-committed cells. Nature 448:553-560.

94. Rousseaux S, et al. 2005. Establishment of male-specific epigenetic information. Gene 345:139-153.

95. Govin J, et al. 2007. Pericentric heterochromatin reprogramming by new histone variants during mouse spermiogenesis. J Cell Biol 176:283294.

96. Pivot-Pajot C, et al. 2003. Acetylation-dependent chromatin reorganization by BRDT, a testis-specific bromodomain-containing protein. Mol Cell Biol 23:5354-5365

97. Loi $\mathrm{P}$, et al. 2002. Nuclei of nonviable ovine somatic cells develop into lambs after nuclear transplantation. Biol Reprod 67:126-132

98. Lai CM, Lai YK, Rakoczy PE. 2002. Adenovirus and adeno-associated virus vectors. DNA Cell Biol 21:895-913.

99. Chung YG, Mann MR, Bartolomei MS, Latham KE. 2002. Nuclearcytoplasmic "tug of war" during cloning: effects of somatic cell nuclei on culture medium preferences of preimplantation cloned mouse embryos. Biol Reprod 66:1178-1184. 